In der Rubrik „Literatur kompakt" werden die wichtigsten Originalarbeiten aus der internationalen Fachliteratur referiert.

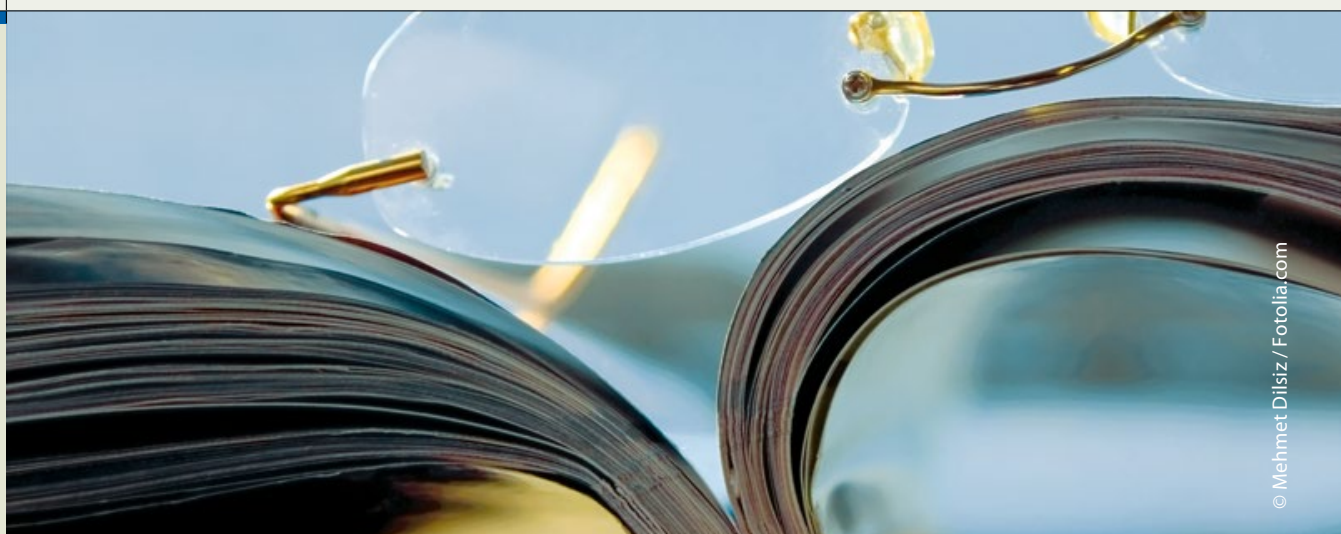

\section{Im Schatten lauert der Tod}

\section{Wer Sonnenbäder zeitlebens meidet, hat zwar ein niedrigeres Risiko Hautkrebs zu entwickeln, stirbt aber dafür früher. Dieses scheinbare Paradoxon haben schwedische Forscher näher untersucht und die Todesursachen je nach Sonnenexposition aufgeschlüsselt.}

$V^{\prime}$ or knapp zwei Jahren ergab eine Studie mit der schwedischen MISSKohorte (Melanoma in Southern Sweden), dass Frauen, die sich regelmäßig gesonnt hatten, einerseits ein niedrigeres Sterberisko und andererseits ein erhöhtes Hautkrebsrisiko haben als Frauen, die Sonne weitgehend mieden. In einer Folgestudie wurden nun mittels einer Cox-Regressionsanalyse die Todesursachen abhängig von der UV-Exposition untersucht. Hierzu wurden Daten von 29.518 Schwedinnen der MISSKohorte, die über 20 Jahre begleitet worden waren, ausgewertet und um Störfaktoren bereinigt.
Mortalität wurde in drei Kategorien eingeteilt: kardiovaskuläre Ursachen, Krebs sowie keins von beidem. Das Sonnenverhalten der Frauen wurden ebenfalls in drei Gruppen eingeteilt: in Sonnenvermeider (das Hauptaugenmerk der Studie), Frauen mit moderater Exposition und "Sonnenanbeter".

Ein 50 Jahre alter Nichtraucher, der die Sonne zeitlebens gemieden hat, hat demnach eine um 0,6 Jahre verringerte Lebenserwartung, bei einem 60 -Jährigen ist sie sogar um 1,3 Jahre verringert. Der gleiche Vergleich bei Rauchern ergab kürzere Lebenserwartungen von 1,1 und 2,1 Jahren. Aus der Tatsache,

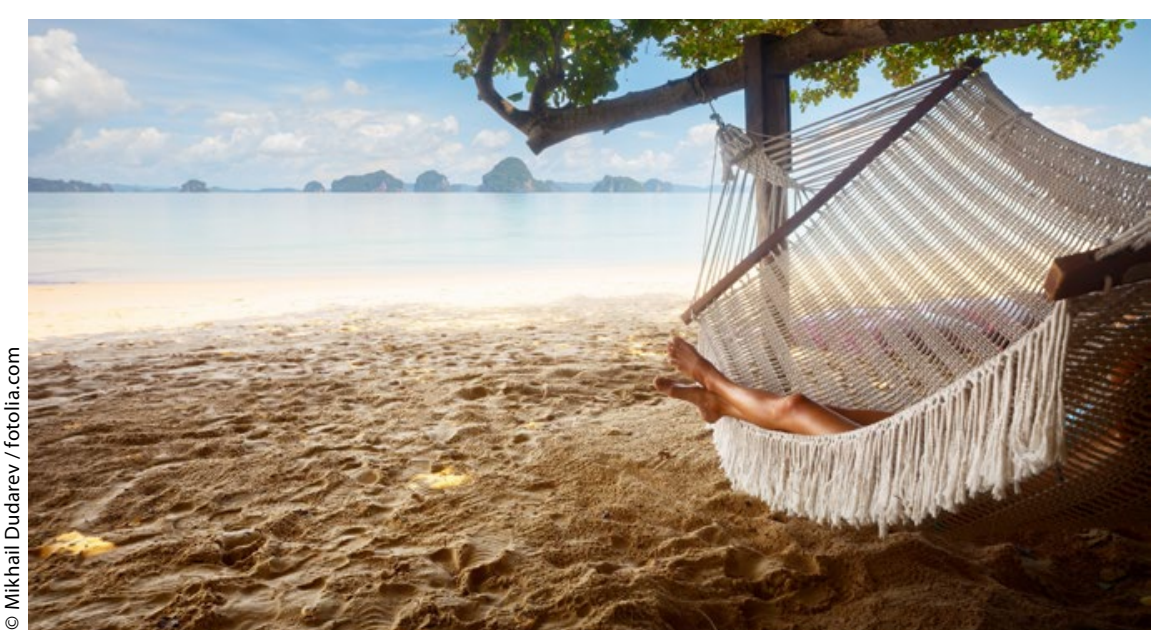

Aufenthalt im Schatten: lebensverlängernd oder lebensverkürzend? dass ein Raucher mit der höchsten Sonnenexposition eine ähnliche Lebenserwartung hat wie ein nicht rauchender Sonnenvermeider, folgern die Autoren, dass Sonne zu meiden ähnlich gesundheitsschädlich ist wie Rauchen.

Im Vergleich zu Frauen, die Sonne mieden und keinerlei Hautkrebs entwickelten (Referenz) hatten sonnenbadende Frauen mit nicht melanozytärem Hautkrebs die geringste Sterbewahrscheinlichkeit. Sowohl in der Melanom- als auch in der Nicht-Melanom-Subgruppe war die Abnahme der Sterbewahrscheinlichkeit sonnendosisabhängig. Gleichzeitig geht die höhere Lebenserwartung von Frauen mit hoher UV-Exposition einher mit einer Abnahme an kardiovaskulären und sonstigen Todesursachen. Demzufolge nimmt dadurch der relative Anteil an Todesfällen durch Krebs zu.

Fazit: Die vorliegende Analyse zeigt, dass UV-Exposition lebensverlängernd zu sein scheint. Ob diese Effekte Vitamin-A-vermittelt sind oder auf einem anderen Mechanismus beruhen, lässt sich anhand dieser Daten nicht erklären. Die Autoren weisen darauf hin, dass bei der Interpretation ihrer Ergebnisse zu beachten sei, dass in Schweden selbst im Sommer oft nur ein UV-Index von 3-5 erreicht werde. Demnach müssten bei Hinweisen zum Sonnen(schutz)verhalten Nutzen und Gefahren sorgfältig abgewogen und an die jeweilige länderspezifische UV-Belastung angepasst werden.

Sebastian Lux

Lindqvist PG et al. Avoidance of sun exposure as a risk factor for major causes of death: a competing risk analysis of the Melanoma in Southern Sweden cohort. J Intern Med. 2016; doi: 10.1111/ joim.12496 